\title{
EPoSS und Smart Anything Everywhere: international vernetzte Technologieplattformen
}

\author{
Rainer Günzler und Stephan Karmann \\ Hahn-Schickard \\ D-78052 Villingen-Schwenningen, Wilhelm-Schickard-Str. 10 \\ rainer.guenzler@hahn-schickard.de
}

\begin{abstract}
Zusammenfassung
Der Beitrag beschreibt international vernetzte Technologieplattformen, ihre Arbeitsweise und konkrete Ergebnisse. Er will dazu motivieren diese Plattformen zu nutzen und mit den Zielen von Firmen, Hochschulen und Forschungseinrichtungen zu verknüpfen. Wie das gelingen kann und welche Vorteile es bringt wird anhand einiger konkreter Beispiele aufgezeigt.
\end{abstract}

Keywords: Sensor,Aktor,Smarte Systeme,Technologieplattform

\section{Technologieplattformen}

Der Begriff „Technologieplattform“ wird in unterschiedlichen Zusammenhängen benutzt. In diesem Beitrag bei den sog. Europäischen Technologieplattformen, den ETPs, für ClusterInitiativen aber auch für technologische „Baukasten-Systeme“. Europäische Technologieplattformen sind Zusammenschlüsse von „Stakeholdern“, die finanzielle und personelle Ressourcen im Bereich Forschung, technologische Entwicklung und Innovation mobilisieren sollen. Sie bedürfen einer formalen Anerkennung durch die Europäische Kommission und spielen eine Schlüsselrolle bei der Definition von europäischen Forschungsprioritäten.

\section{EPoSS, die europäische}

Technologieplattform für Smarte Systeme

Die für den Bereich Sensoren wohl wichtigste europäische Technologieplattform ist EPoSS (www.smart-systems-integration.org). „Smarte Systeme" kombinieren kognitive, sensorische und aktorische Funktionen mit Datenübertragung und Energiemanagement in einer integrierten Weise. „Smart Systems Integration" steht für eine Weiterentwicklung der Mikrosystemtechnik, allerdings bezieht sich die Definition des Begriffes nicht auf Technologien oder Abmessungen, sondern auf Funktionalitäten.

EPoSS wurde von einer Vielzahl an der Thematik interessierter Personen und Insti- tutionen aufgebaut und ist ein eingetragener Verein nach deutschem Recht.

In den Arbeitsgruppen von EPoSS werden Erfahrungen ausgetauscht und z.B. regelmäßige Updates der Roadmap und der Strategischen Forschungsagenda (SRA) erstellt. Die Plattform hat sich im vergangen Jahrzehnt eine gute Stellung im Reigen der Europäischen Technologieplattformen erarbeitet. Inzwischen ist EPoSS Partner der Gemeinsamen Unternehmung ECSEL (Joint Undertaking Electronic Components and Systems for European Leadership), einer Rechtsform, die selbst Projekt-Ausschreibungen durchführt. Durch die Mitgestaltung der jährlichen Arbeitsprogramme hat EPoSS Einfluss auf die Inhalte dieser Ausschreibungen.

Smart Anything Everywhere, eine junge Initiative der Europäischen Kommission

Die Europäische Kommission will insbesondere kleine und mittlere Unternehmen (KMU) in die Lage versetzen, die Herausforderungen des digitalen Wandels zu meistern. Dazu hat sie mehrere Initiativen ins Leben gerufen. Eine tragende Säule ist die sog. Smart Anything Everywhere Initiative (www.smartanythingeverywhere.eu). Sie bildet ein Dach für ein Dutzend Projekte mit einem Umfang von bisher insgesamt ca. $60 \mathrm{M} €$, die das gemeinsame Ziel haben, durch regional fokussierte „Digital Innovation Hubs“ (DIHs) die Unternehmen bei der Digitalisierung zu unterstützen. Die Projekte der SAE Initiative 
nutzen dabei ein neues Förderinstrument, die „Innovation Action“ (IA). Hier wird nicht elementare Forschung und Entwicklung gefördert, sondern gezielt in marktnahe Ergebnisse investiert, die im Laufe des Projektes erreicht und möglichst auch kommerzialisiert werden sollen. Dazu können die Projekte das Instrument "Cascade Funding" nutzen, also eigene Ausschreibungen veröffentlichen. Dies ermöglicht kleinere „Unterprojekte“, um im Verbund aus Unternehmen und Forschungseinrichtungen neue Anwendungen im Bereich Digitalisierung umzusetzen. Ein wesentlicher Vorteil dabei ist, dass die Unternehmen selbst keinen Vertrag mit der Kommission eingehen müssen. Diesen Vertrag schließen lediglich die Innovation Hubs, die sich als Projektpartner der jeweiligen IA um alle bürokratischen Erfordernisse wie Berichterstattung und Abrechnung kümmern.

\section{Das Projekt SMARTER-SI}

Eine dieser Innovation Actions ist SMARTERSI (www.smarter-si.eu), ein Projekt, das in Arbeitsgruppen von EPoSS entstanden ist. Die dem Projekt zugrundeliegende Idee ist, dass wirtschaftsnahe Forschungseinrichtungen Kleinserienproduktionen für KMU übernehmen, wenn sich daraus ein Vorteil für Unternehmen wie Institute ergibt.

Ein Vorteil für KMU ist dann gegeben, wenn sie z.B. Sensorsysteme einsetzen möchten, die kommerziell nicht verfügbar sind, und die benötigten Stückzahlen so gering sind, dass sie von kommerziellen Herstellern (Foundries) nicht bereitgestellt werden können.
Für Forschungseinrichtungen können gerade solche Kleinserien attraktiv sein! Etwa dann, wenn sie in vorausgegangenen F\&E Projekten Demonstratoren oder Prototypen erarbeitet haben, die in einem schon hohen Technologiereifegrad vorliegen und nun in neuen Anwendungsfeldern eingesetzt und kommerzialisiert werden können. Ggf. müssen vielleicht Schnittstellen angepasst, andere Formfaktoren gefunden oder Software ergänzt werden - ein Aufwand, der aber in jedem Fall sehr viel geringer ist als eine Neuentwicklung. Die Aufgabe besteht also darin, das Angebot an vorhandenen Technologiebausteinen („building blocks") auf Seiten der Institute mit den Anforderungen auf Seiten der KMU in Deckung zu bringen.

Diese sehr anspruchsvolle Aufgabe ist im Projekt SMARTER-SI für insgesamt 11 Anwendungsbeispiele gelungen. Im Ergebnis können insgesamt $18 \mathrm{KMU}$ aus unterschiedlichen Europäischen Ländern jeweils einige Dutzend smarte Systeme zur Verfügung gestellt werden, so dass die Unternehmen entsprechende Betatests mit ihren Kunden durchführen können. Die Foundry-Plattform erniedrigt die Eintrittsschwelle für $\mathrm{KMU}$ und eröffnet den schnellen Zugang zu verfügbaren Technologiebausteinen, europaweit. Einige der avisierten Produkte sollen schon wenige Monate nach Projektende auf den Markt kommen. Damit werden für die KMU zusätzliche Umsätze generiert, die bereits in den ersten drei Jahren nach dem Projekt die investierten Fördermittel übersteigen.

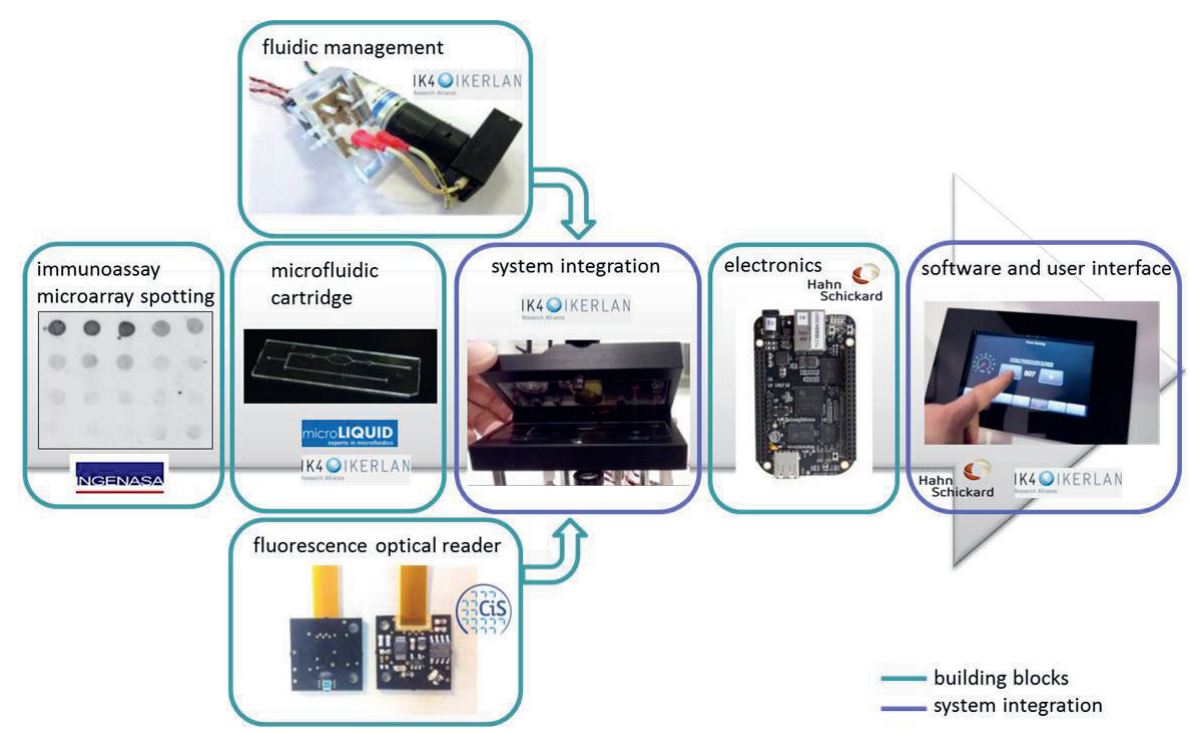

Abb. 1: Technologiebausteine für ein Point-ofCare Testing Gerät. 
Abbildung 1 zeigt die Technologiebausteine für ein modulares Multi-Parameter Point-of-Care (PoC) Testing Gerät. Der Begriff Technologiebausteine wird pragmatisch verwendet. $\mathrm{Er}$ kann sowohl für eine sensitive Schicht, einen Sensor-Chip, eine Auswerteelektronik, eine Benutzer-Schnittstelle oder eine Software stehen. Eine möglichst reibungslose Integration der verschiedenen Technologiebausteine erfordert präzise Beschreibungen der Funktionalitäten und Schnittstellen.

Abbildung 2 zeigt ein assembliertes PoC Gerät, das ohne spezielle Vorkenntnisse z.B. zur Detektion von Mikrotoxinen in Lebensmitteln verwendet werden kann. Als weitere Beispiele werden in Abbildung 3 eine smarte Mikrotiterplatte zur Charakterisierung biologischer Substanzen und in Abbildung 4 ein modulares System für Atemanwendungen gezeigt, mit Teilen die wiederverwendet werden und solchen, die nur einmal benutzt werden, eine hochintegrierte und miniaturisierte Sensorik für verschiedene medizinische und well-being Anwendungen.

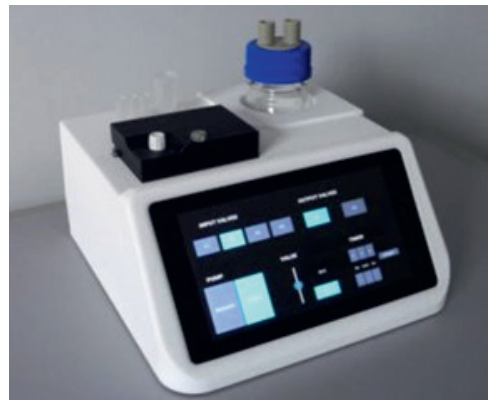

Abb. 2: Modulares Multi-Parameter Point-ofCare Testing Gerät.

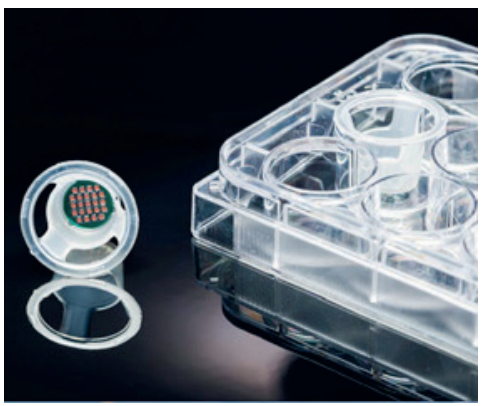

Abb. 3: Smarte Mikrotiterplatte zur Bestimmung transepithelialer elektrischer Widerstände.

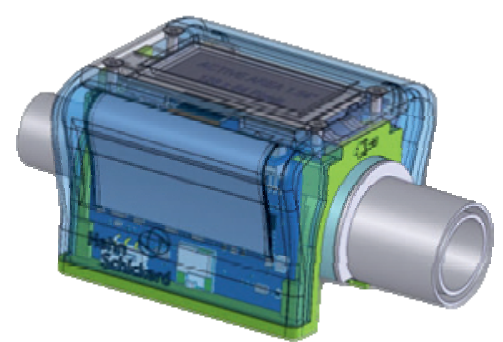

Abb. 4: Sensorsystem zur Bestimmung von Atemfluss und $\mathrm{CO}_{2}$ Konzentration.

\section{Zusammenfassung und Ausblick}

In Arbeitsgruppen der europäischen Technologieplattform EPoSS wurde die Idee zu dem Projekt SMARTER-SI entwickelt. SMARTER$\mathrm{SI}$ ist ein Testumfeld für die Produktion kleiner Stückzahlen für KMU durch eine kooperative Foundry, basierend auf marktnahen Ergebnissen - sogenannten Technologiebausteinen - von Forschungseinrichtungen. SMARTER-SI ist sozusagen eine in einer Europäischen Technologieplattform entstandene „technische Technologieplattform“. Die im Projekt bisher gewonnenen Erfahrungen und die erreichten Ergebnisse stimmen optimistisch und daher arbeitet das Konsortium an der Analyse von Möglichkeiten, um eine Nachhaltigkeit sicherzustellen, mit aber auch ohne Fördermittel.

Die Beschreibung von Funktionalitäten und Schnittstellen der Technologiebausteine wie auch deren Reifegrad haben sich als wesentlich erwiesen. Deshalb arbeitet das Konsortium von SMARTER-SI an einer entsprechenden Taxonomie, an Möglichkeiten zur Beschreibung von Kategorien und zur Klassifizierung, Vorstufen zur einer bisher im Wesentlichen nicht existenten Standardisierung von Bestandteilen Smarter Systeme.

SMARTER-SI wird gefördert durch das europäische Forschungsrahmenprogramm HORIZONT 2020 unter der Zuwendungsvereinbarung Nr. 644596 und durch das Schweizer Staatssekretariat für Bildung, Forschung und Innovation (SBFI), Vertragsnummer 15.0085 . 